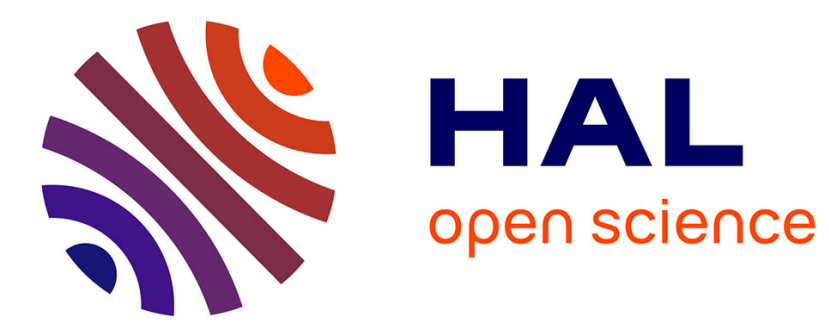

\title{
Immigration, migration et libre circulation dans la construction de l'Europe
}

\author{
Adrian Favell
}

\section{To cite this version:}

Adrian Favell. Immigration, migration et libre circulation dans la construction de l'Europe. Politique européenne, 2010, 2 (31), pp.33-64. 10.3917/poeu.031.0033 . hal-01024114

HAL Id: hal-01024114

https://hal-sciencespo.archives-ouvertes.fr/hal-01024114

Submitted on 15 Jul 2014

HAL is a multi-disciplinary open access archive for the deposit and dissemination of scientific research documents, whether they are published or not. The documents may come from teaching and research institutions in France or abroad, or from public or private research centers.
L'archive ouverte pluridisciplinaire HAL, est destinée au dépôt et à la diffusion de documents scientifiques de niveau recherche, publiés ou non, émanant des établissements d'enseignement et de recherche français ou étrangers, des laboratoires publics ou privés.

\section{(ㅇ)(1) $\$$}

Distributed under a Creative Commons Attribution - NonCommercial - NoDerivatives 44.0 


\title{
IMMIGRATION, MIGRATION ET LIBRE CIRCULATION DANS LA CONSTRUCTION DE L'EUROPE
}

\author{
Adrian Favell \\ L'Harmattan | Politique européenne
}

$2010 / 2-n^{\circ} 31$

pages 33 à 64

ISSN 1623-6297

Article disponible en ligne à l'adresse:

http://www.cairn.info/revue-politique-europeenne-2010-2-page-33.htm

Pour citer cet article :

Favell Adrian, « Immigration, migration et libre circulation dans la construction de l'Europe », Politique européenne, 2010/2 n³1, p. 33-64.

Distribution électronique Cairn.info pour L'Harmattan.

(C) L'Harmattan. Tous droits réservés pour tous pays.

La reproduction ou représentation de cet article, notamment par photocopie, n'est autorisée que dans les limites des conditions générales d'utilisation du site ou, le cas échéant, des conditions générales de la licence souscrite par votre établissement. Toute autre reproduction ou représentation, en tout ou partie, sous quelque forme et de quelque manière que ce soit, est interdite sauf accord préalable et écrit de l'éditeur, en dehors des cas prévus par la législation en vigueur en France. II est précisé que son stockage dans une base de données est également interdit. 


\section{IMMIGRATION, MIGRATION ET LIBRE CIRCULATION DANS LA CONSTRUCTION DE L'EUROPE}

Bien que les Européens semblent sédentaires en comparaison des habitants de l'Amérique du Nord, l'histoire européenne a connu d'incessants et d'importants mouvements de populations qui continuent aujourd'hui. Qu'en est-il du rôle de la mobilité spatiale dans toutes ses formes dans la construction européenne? Cet article répond à cette question en analysant les trois types de migrants dans l'Europe contemporaine: les populations issues des vagues migratoires de l'après-guerre et les nouveaux migrants des pays tiers, les "libres circulants" ou "Eurostars" utilisant le droit à la libre circulation inscrit dans le Traité à la libre circulation, et enfin les ressortissants des pays de l'Europe centrale et orientale entrés dans l'Union européenne (UE) en 2004 et 2007 qui peuvent désormais vivre et travailler dans d'autres pays de l'UE. On peut dire qu'un nouveau système migratoire en Europe se met en place, qui repose sur un marché de travail secondaire exploitant les libres circulants de l'est, une ouverture à l'Est qui a permis une politique de plus en plus sécuritaire de fermeture au Sud avec une logique raciale claire.

\section{Immigration, migration, free movement and the making of Europe}

Despite the image of European populations as sendentary in comparison to the highly mobile populations of North America, population movements have played a recurrent role in the history of Europe, up to and including the present day. How then does spatial mobility in all its forms fit into the European integration process? This article answers this question by contrasting the three principle types of migrants in Europe today: the traditional, post war immigration of "ethnically" distinct populations from non-European sources, the "free movers" or "Eurostars" living and working as foreigners within Europe via the use of EU free movement rights, and the citizens from the new East and Central European member states who have gained access to the West European labour market as a result of the EU enlargements of 2004 and 2007. It argues that a new European migration system, dependent on the secondary labour market exploitation of new East-West movers, is in the making, an opening to the East that has enabled a stricter attempt to close doors to immigration from the South and elsewhere along clearly racialised lines.

politique européenne, $\mathrm{n}^{\circ} 31,2010$, p. 33-64. 
D'un point de vue historique, l'Europe s'est construite, déconstruite et reconstruite par le biais des mouvements de populations ${ }^{1}$. Malgré l'image plutôt sédentaire et socialement peu mobile actuellement prêtée aux Européens, notamment en comparaison avec les modèles nordaméricains de populations à forte mobilité spatiale et sociale, l'Europe contemporaine est le résultat d'un creuset de populations qui se sont déplacées aux niveaux local, régional et international au cours des siècles.

Dans cet article, j'aborde l'impact important des migrations sur l'identité européenne, en faisant le lien entre les analyses historiques du phénomène et les schémas émergents qui façonnent l'Europe en tant que nouvel espace régional de migration et de mobilité. Ma contribution montre dans quelle mesure l'Europe se construit et se reconstruit par les migrations. Il s'agit peu, en fait, de la façon dont les gens s'identifient à l'Europe : en réalité, la plupart des migrations contribuent plutôt au développement d'un sentiment anti-européen. L'impact des migrations se situe plutôt au niveau territorial et surtout d'un point de vue structurel. Ceci est plus difficile à mettre en lumière à partir d'une vision purement culturelle de la question européenne. Une fois esquissé le rôle des mouvements de population dans la construction et la reconstruction de l'Europe d'un point de vue historique, je présenterai plus en détail les trois types de migration/mobilité les plus marquants du continent aujourd'hui : en premier lieu, l'immigration traditionnelle « extra-européenne » qui se poursuit, et qui concerne des ressortissants des pays tiers dans les États nations européens ; en deuxième lieu, l'émergence, numériquement faible mais symboliquement importante, des migrations d'une nouvelle " élite » intra-européenne, qui concerne des citoyens européens profitant de leur liberté de circulation au sein de l'UE; et en troisième lieu, les flux de migrants suivant une trajectoire Est-Ouest - qui se situent quelque part entre les deux autres formes de migration $-s$ 'inscrivant dans les processus d'élargissement de l'UE ayant donné lieu aux adhésions de douze pays en 2004 et en 2007. La meilleure manière d'appréhender le caractère distinctif de l'Europe comme entité régionale - et, ainsi, son

1 Cet article est une traduction du chapitre "Migration, immigration and free movement in the making of Europe ", publié in Jeffrey T. Checkel et Peter J. Katzenstein (dir.), European Identity, Cambridge, Cambridge University Press, 2009, p. 167-189 [publié dans cet ouvrage avec la permission des auteurs]. Il constitue une nouvelle présentation des idées développées initialement dans Favell (2003a). Il a été traduit par Cyril Le Roy et édité par Virginie Guiraudon, qu’ils en soient ici vivement remerciés. 
identité économique et territoriale - est de la comparer brièvement à nouveau aux États-Unis et à l'Amérique du Nord comme espace régional de migration comparable, mais structuré différemment, thème sur lequel je reviendrai en guise de conclusion.

\section{Mouvements de population dans la construction et la déconstruction de l'Europe}

Il n'est pas rare que les citoyens européens se voient prêter une prédisposition naturelle à l'immobilité. L'Europe est généralement vue comme un patchwork de cultures profondément " ancrées ", avec des langues et des pratiques sociales fièrement préservées, dressant le tableau d'un continent constitué de peuples aux racines inébranlables, avec de fortes identités nationales et locales, peu sensibles aux efforts des institutions européennes - ou à la mondialisation - pour faire évoluer leur façon de penser. On la considère également comme un continent en grande partie hostile aux nouveaux immigrants, ayant des difficultés à intégrer les diverses minorités ethniques et raciales, pourtant faibles numériquement, qui parviennent à y entrer.

Comme souvent, la situation aux États-Unis est invoquée pour souligner ce contraste. Si l'UE peut être rapidement désignée comme une sorte d'États-Unis d'Europe, les chiffres laissent peu de doute. Alors que $12 \%$ des Américains sont nés à l'étranger (Batalova et Lowell, 2006), moins d'un Européen sur cinquante réside hors de son pays de naissance, et même les migrations au sein des États nations européens sont moins importantes que les migrations inter-États aux États-Unis, représentant $22 \%$ de la population en Europe comparés à $33 \%$ aux États-Unis (Fondation européenne pour l'amélioration des conditions de vie et de travail, 2006). La société européenne peut ainsi être vue comme le produit de cultures enracinées historiquement ; l'Amérique s'est quant à elle incontestablement construite par l'immigration et le mélange des nouveaux arrivants. Malgré des résistances fluctuantes à la nouvelle immigration, le nombre de personnes d'origine immigrée récente sur les deux ou trois dernières générations, qui représentent plus de la moitié de la population dans certains États comme la Californie est plus important aux États-Unis que dans les États européens. Ce n'est qu'après le 11 septembre où l'Europe se ferma un moment que les arrivées dans l'Europe des 15 furent plus importantes qu'aux États-Unis : 
en 2003, deux millions de personnes migrèrent légalement (Eurostat, 2009, p. 54). Cependant, l'attractivité des États-Unis pour des nouvelles générations d'individus ambitieux et talentueux du monde entier reste incomparable avec celle de l'Europe comme force économique globale : deux tiers des migrants avec une formation dans le secteur tertiaire en provenance des pays en développement choisissent l'Amérique comme destination, avec des bénéfices considérables pour l'économie américaine (Peri, 2005). En résumé, il apparaît donc que les Américains sont disposés à bouger et à accepter les migrants, contrairement aux Européens.

Un peu de recul par rapport à cette hypothèse révèle toutefois rapidement son inexactitude d'un point de vue historique (voir aussi Recchi, 2006). Après tout, l'Amérique a en grande partie été peuplée par des Européens qui ont traversé l'océan pour immigrer, avant de se déplacer encore sur de vastes territoires. Considérée à moins courte vue, l'Europe reste et a toujours été un continent de flux migratoires. Les débuts de l'Europe moderne - l'Europe telle qu'elle est célébrée partout par les nationalistes en termes de folklore enraciné culturellement (Hobsbawm, 1983 ; Anderson, 1991) - étaient effectivement caractérisés par un patchwork de mobilité circulaire, saisonnière et professionnelle bien avant l'industrialisation. Les révolutions ont ensuite tout changé, chassant les paysans de leurs terres, déstructurant les communautés rurales, provoquant l'entassement de nouvelles classes sociales dans des villes en expansion et créant les routes économiques de mobilité reliant l'ensemble de l'Europe, puis du monde, dans un nouveau système impérialiste et capitaliste (Moch, 2003 ; Bade, 2000 ; Hobsbawm, 1987). Concrètement, cela se traduisait par des flux migratoires continuels. La fin du XIXe siècle est marquée par des mouvements inédits de travailleurs qui passent les frontières nationales et de migrants et colons qui traversent les mers pour s'installer dans le nouveau monde (Hatton et Williamson, 1998). Les Européens allèrent partout.

Les raisons pour lesquelles ces faits sont oubliés dans l'image de l'Europe sédentaire d'aujourd'hui se trouvent évidemment dans les guerres du XXe siècle qui ont porté un coup d'arrêt à l'essentiel de ces migrations. Les États nations ont fini par devenir partout la forme dominante d'organisation sociale, institutionnalisant un pouvoir centré autour de l'État pour contenir des populations explosives, utilisant le service militaire, la citoyenneté et les droits sociaux au nom de l'identité nationale, pour créer des distinctions entre les membres de la communauté politique et ceux de 
l'extérieur, et fixant géographiquement les populations (Torpey, 2000 ; Mann, 1993). Ceci devient ensuite la topographie politique légitime du monde moderne, laissant de nombreux groupes ethniques du mauvais côté des frontières ou dans des situations de relégation sociale, populations résiduelles sans État dans une Europe désormais complètement nationalisée. Ceci engendra un désastre - la Shoah - dont le continent portera pour toujours les cicatrices, ainsi qu'un horrible aprèsguerre entraînant encore plus de déplacements forcés de populations vers l'Est et l'Ouest. Les Européens furent une nouvelle fois déplacés, à la recherche d'une solution politique stable qui pourrait une fois pour toutes régler le problème des frontières ethniques et idéologiques de ce que certains appelaient la «zone de déflagration » en Europe centrale et orientale (Mazower, 1998 ; Brubaker, 1996 ; Mann, 2005). L'Europe abandonna ses empires et le rideau de fer créa une nouvelle barrière matérielle et psychologique pratiquement imperméable, gelant les déplacements entre Est et Ouest et interrompant littéralement tous les déplacements terrestres latitudinaux et les interactions qui, pour les historiens-géographes, avaient constitué sur la longue durée la manne civilisatrice du continent (Diamond, 1997).

À l'Ouest, les structures généreuses de l'État-providence de l'aprèsguerre - sorte de forme démocratique libérale du nationalisme socialisé qui prévalait sur l'ensemble du continent jusqu'en 1970 (Mazower, 1998) - ont ancré les populations nationales comme jamais auparavant. Les puissances affaiblies d'Europe occidentale ont fini par renaitre sur le plan économique, mais cela s'est produit en faisant alors appel à l'immigration pour répondre à leurs besoins en main-d'œuvre, d'abord via une première vague de travailleurs originaires du Sud voisin migrant vers le Nord (Italiens, Espagnols, Portugais et Yougoslaves), puis - lorsque ces mouvements se sont également taris - via une immigration d'une ampleur sans précédent en provenance des anciennes colonies et des dépendances extra-européennes (en particulier de Turquie, d'Afrique du Nord, d'Afrique Centrale, des Antilles, d'Asie du Sud et d'Indonésie). Ceci a évidemment produit un mélange encore plus détonant d'origines ethniques et de diversité culturelle dans un continent réticent (Castles et Miller, 2003).

Une représentation historique de la carte des mouvements de population européens, aussi difficile qu'elle puisse être, est nécessaire pour engager toute discussion sur la place des migrations aujourd'hui dans la construction de l'identité européenne. Cette carte est difficile à établir 
(voir aussi King, 2002). La fin des années 1970 est censée avoir marqué l'arrêt de l'immigration en provenance des anciennes colonies et de l'immigration de travail, limitant les possibilités d'entrée au regroupement familial et à l'asile. Les populations immigrées étaient censées s'être installées et être intégrées par l'acquisition de la nationalité et de la citoyenneté, transformant ce faisant à contre cœur des sociétés nationales plus ou moins culturellement homogènes en sociétés multiculturelles.

Les années 1980, et encore plus les années 1990, ont une nouvelle fois tout bouleversé. Une vague de "nouvelles migrations " a mélangé encore plus le continent (Koser et Lutz, 1998). La mondialisation de l'économie s'est accompagnée de la libéralisation des sociétés post-industrielles, débouchant sur une nouvelle économie duale de service reposant de manière importante sur la disponibilité de main-d'œuvre étrangère à bas coût (Sassen, 2001 ; Piore, 1989). Les systèmes de transport organisés à l'échelle mondiale ont facilité le mouvement vers l'Europe depuis des régions de plus en plus diverses et imprévisibles (Held et al., 1999). Comme en Amérique, les classes ouvrières européennes ne veulent plus assurer les tâches correspondant aux « 3D jobs " (" dirty, dangerous and dull jobs ") - c'est-à-dire les emplois sales, dangereux et abrutissants qui peuvent être affectés à des étrangers plus motivés et moins bien payés. Comme dans les autres parties du monde, les migrations se sont considérablement féminisées, les femmes des pays en développement occupant les emplois dans le secteur des soins aux personnes et de l'aide à domicile dans les pays développés. Les demandes d'asile, phénomène autrefois essentiellement symbolique permettant à des nombres réduits de réfugiés politiques de s'échapper vers l'Ouest, ont vu leur nombre fortement augmenter, l'Europe récupérant les morceaux des déchirements provoqués par les nombreuses guerres civiles ou régionales ; l'asile est également devenu en réalité une filière de migration de travail. L'Europe était censée devenir une forteresse, alors que depuis le début des années 2000, c'est l'inverse qui se produit (Favell et Hansen 2002).

En sus de ces nouvelles formes d'immigration, de nouvelles migrations intracommunautaires à l'UE se sont développées. L'Union européenne s'est construite autour des quatre libertés, dont la libre circulation des personnes. Cela fait maintenant des dizaines d'années que les ressortissants des premiers États membres de l'UE bénéficient de ces droits. Les mouvements d'Européens de l'Ouest n'ont jamais été très 
importants par leur nombre, mais ils revêtent un caractère symbolique fort. Pour chaque Européen qui déménage pour travailler et s'installer librement dans un État membre voisin dans l'Union européenne, on compte un nombre beaucoup plus important se déplaçant temporairement en tant qu'étudiant, consommateur, travailleur transfrontalier, ou même retraité. Si on ajoute à cela la redéfinition permanente des frontières géographiques de l'Union, conséquence des élargissements successifs, la possibilité d'un nouveau type de migration en Europe (" libre circulation ») semble en mesure de déconstruire et reconstruire le patchwork existant de sociétés nationales, qui avait, avec plus ou moins de succès, utilisé l'Union européenne pour sauver le modèle de l'État nation européen dans la période de l'après-guerre (Milward, 2005). Les personnes les plus visibles parmi les Européens utilisant leur droit à la libre circulation (les " libres circulants " ou " euromobiles ") sont bien sûr les ressortissants des pays d'Europe de l'Est et d'Europe centrale, très mobiles dans l'espace et, dans certains cas, sur l'échelle sociale, profitant d'un espace européen qui leur est à nouveau entièrement accessible. Mais étant donné la logique de construction concentrique de l'UE vers son voisinage extérieur, les "libres circulants" viendront également d'Ukraine, de Turquie et du Maroc dans le futur.

La combinaison de ces phénomènes donne un cadre assez complexe pour l'évaluation de l'impact des mouvements de population sur l'identité européenne. Comme je le montrerai par la suite, en analysant l'impact de ces différentes migrations sur la construction et la déconstruction européenne, on peut voir que l'Europe peine à maintenir des distinctions entre les trois groupes de migrants. Je dresserai ici les grandes lignes de ce futur probable, avant d'étudier ce scénario de manière plus approfondie dans le reste de l'article. ${ }^{2}$

Un premier type de migration (constituée de ressortissants des pays tiers d'origine modeste en provenance d'Afrique et d'Asie, dans la mesure où ils peuvent être distingués comme tels) suit actuellement un processus, marqué par de nombreux conflits sociaux et politiques, dans lequel on retrouve les méthodes traditionnelles de gestion des travailleurs immigrés de l'après-guerre originaires des anciennes colonies. La question de ces immigrants continue d'être principalement traitée au

2 Fin 2007, l'Europe des 27 accueillait 29,1 millions d'étrangers dont 10,6 millions de migrants intracommunautaires (Recchi et Triandafillydou, 2009). 
niveau des sociétés nationales, et non au niveau de l'Union européenne. Les dernières années ont été marquées par le retour de politiques nationalistes en matière d'intégration dans toute l'Europe, qui alimentent de plus en plus les politiques de fermeture symbolique anti-UE et antimondialisation. Cependant, en Europe comme ailleurs, ces politiques d'immigration, au visage souvent détestable, ne sont pas en phase avec la réalité économique. La nostalgie de sociétés maîtrisées, sans problème d'identité culturelle, fondées sur la citoyenneté et la nation, fait mauvais ménage avec un marché du travail dual et mondialisé dans des économies reposant sur un secteur des services dirigé par des multinationales, qui ont besoin d'une armée de réserve pratiquement infinie de main-d'œuvre étrangère flexible.

Les expériences vécues par le second groupe de migrants, situé à l'extrême opposée de l'échelle sociale (Européens de l'Ouest jouissant de la libre circulation, que j'appelle les « Eurostars»), racontent une histoire différente sur l'Europe d'aujourd'hui. Sans surprise, ils sont le reflet le plus enthousiasmant d'une Europe cosmopolite, postnationale. Toutefois, même si tous les obstacles formels à la migration ont été levés, leurs mouvements restent soumis à des restrictions et à une résistance qui soulignent la résilience des cultures nationales, jusque dans les lieux les plus structurellement mondialisés et internationaux (Londres et Amsterdam étant utilisés comme exemples).

Un troisième groupe de migrants (constitué des nouveaux immigrés d'Europe de l'Est) est le plus équivoque des trois. Doivent-ils être considérés comme des "Eurostars » en devenir, comme le prédisent la théorie néofonctionaliste de l'intégration européenne et les théories économiques ? Ou sont-ils encore plutôt semblables aux travailleurs immigrés de l'après-guerre, et plus vraisemblablement traités comme tels ? J'apporterai une réponse positive aux deux questions. Ils participent à la construction d'un nouvel espace européen de circulation et réalisent une nouvelle idée de la citoyenneté européenne, mais ils sont dans le même temps renvoyés à des rôles économiques affectés traditionnellement aux immigrés non-européens dans la période de l'après-guerre. Là se trouve un des facteurs clés de l'évolution à venir. Une sorte de forteresse européenne pourrait être construite sur le dos de cet élargissement et de cette ouverture ambigus de l'Europe à l'Est. La tentation d'appliquer une logique ethnique existe aujourd'hui en Europe. L'ouverture aux populations de l'Est pourrait permettre la fermeture 
véritable de l'Europe au Sud, satisfaisant un besoin structurel pour lequel l'Europe occidentale avait historiquement fait appel à des migrants en provenance des colonies et de pays en développement aux sociétés et aux cultures plus éloignées. Les distinctions ethniques et culturelles pourraient servir à réaliser ce que béton, surveillance électronique et barbelés sont incapables de faire.

\section{Les trois migrations en Europe}

Je voudrais à présent examiner de manière plus détaillée chacun des trois types de migrations identifiées ci-dessus : immigrants nonEuropéens, « Eurostars » d'Europe occidentale et bénéficiaires post-élargissement de la libre circulation Est-Ouest. Il est essentiel de les distinguer de manière analytique avant de montrer comment les nouvelles dynamiques migratoires brouillent nombre de ces soi-disant distinctions évidentes.

\section{Im (migrants) « classiques" non-européens}

Les nouvelles formes de migration et de mobilité ont modifié le contexte des mouvements de population en Europe, mais le scénario dominant en Europe aujourd'hui reste celui de l'immigration classique postcoloniale des travailleurs immigrés. Les économies européennes génèrent encore incontestablement une forte demande en travailleurs migrants, ainsi qu'une offre qui résulte d'une image séduisante (suffisamment attractive en regard des coûts et de l'incertitude qui entrent dans les calculs des migrants qui tentent leur chance depuis l'Afrique ou l'Asie). Le changement de scénario se trouve dans la diversité toujours plus grande des origines des migrants actuels, comprenant désormais des pays avec des liens coloniaux faibles ou inexistants avec les pays de destination. Les générations antérieures d'immigrants postcoloniaux pouvaient au moins compter sur un lien symbolique avec les destinations métropolitaines, qui allaient souvent de pair avec une relative socialisation dans la langue et la culture du pays. De nos jours, on peut constater un égal éparpillement des pays d'origine et des destinations des migrants, ce qui accentue les tensions émergeant au plan politique autour des questions migratoires dans les sociétés de destination. Par exemple, le contexte d'accueil des migrants en provenance des Antilles 
britanniques au cours des années 1960 est radicalement différent de celui que connaissent les Sri Lankais et les Kurdes arrivant au Danemark aujourd'hui. Les systèmes d'intégration nationaux fonctionnent de moins en moins bien face aux nouvelles migrations.

De plus, les canaux de migration aujourd'hui sont organisés de manière beaucoup plus « horizontale " qu’à l'époque de relative planification des migrations postcoloniales et du recrutement de travailleurs immigrés. Parmi les systèmes de migration qui ont vu le jour, certains sont caractérisés par une auto-organisation interne très particulière : vendeurs de rue sénégalais en Italie (Riccio, 2001), travailleurs domestiques capverdiens en Italie et en Espagne (Andall, 1998), migrants chinois en Angleterre et en France (Benton et Pieke, 1998), immigrés du MoyenOrient en Scandinavie (Diken, 1998), etc. Pour l'essentiel, cependant, les groupes de migrants les plus nombreux (en provenance de Turquie et du Maroc) sont organisés autour de systèmes de migrations plutôt prévisibles et continus qui sont le fruit d'une longue histoire et de connexions aisées avec une série de pays (pour certaines sources, voir Lesthaege, 2000 ; Kastoryano, 1993 ; Bousetta, 2000 ; Phalet et al., 2000).

Comme tout le monde sait, ces diverses immigrations se sont traduites de manière visible par l'arrivée de personnes à peau noire, brune ou jaune en Europe, y compris dans les endroits les plus improbables. Les questions du multiculturalisme et des conflits interethniques, qui étaient auparavant limitées aux anciennes puissances coloniales comme la Grande-Bretagne et la France, se posent désormais dans tous les pays d'Europe occidentale et de plus en plus en Europe de l'Est et en Europe centrale. Étant donné que la majorité de ces nouveaux immigrants sont originaires de pays essentiellement musulmans, cette dimension (que ce soit en termes de pratique religieuse ou simplement de culture d'origine) est devenue la question déterminante en matière d' "identité " européenne la plus souvent associée à l'immigration aujourd'hui (voir aussi Byrnes et Katzenstein, 2006).

Cette question est bien sûr otage des enjeux géopolitiques plus larges liés à la " guerre contre la terreur ", également soumise à des points de vue réducteurs et provocateurs sur le soi-disant " choc des civilisations" (Huntington, 1996). Il est maintenant difficile de dépasser la rhétorique pour évaluer dans quelle mesure ces nouvelles migrations et les problèmes multiculturels différents de ceux de la "vieille " immigration des années 1950 et 1960. En effet, la trajectoire de ces migrants qui constituent 
des " minorités visibles " dans la société européenne est relativement familière. D'un point de vue général, ils constituent une caractéristique structurelle importante de toutes les sociétés européennes d'après-guerre. Les effets ressentis dans les grands pays d'accueil d'immigrés des anciennes colonies et de main-d'œuvre étrangère se retrouvent de manière similaire dans les pays d'Europe du Nord accueillant des demandeurs d'asile puis, ensuite, en Europe du Sud. Chaque État nation a dû faire face à des "questions multiculturelles " similaires, bien qu'à des moments et avec des acuités politiques différentes (Favell, 2001 ; Joppke et Morawska, 2003). L'Europe dans son ensemble est devenue un continent d'immigration et (plus difficilement) une terre d'islam, mais les processus politiques et sociaux engendrés par ces questions ont été partout abordés sous un angle essentiellement national, pour devenir maintenant partout une question centrale de politique intérieure, de la Grande-Bretagne au Danemark en passant par les Pays-Bas et l'Espagne.

L'immigration est donc certes une question européenne, mais les politiques d'immigration restent encore essentiellement nationales in locus. La coopération au niveau européen a eu des effets, en particulier en matière de politiques de contrôle des frontières et d'entrée sur les territoires, mais l'UE a eu peu d'impact sur les politiques ou processus d'installation d'immigrants. Le problème essentiel pour ces immigrants non-européens (les " ressortissants de pays tiers » selon leur désignation dans le jargon de l'UE) consiste à obtenir une citoyenneté officielle et un statut reconnu au niveau national : ils n'ont pas la citoyenneté européenne, bien qu'ils soient résidents permanents. La naturalisation dans le pays d'adoption constitue le chemin le plus sûr pour devenir européen, mais une naturalisation réussie passe inévitablement par un processus national (Hansen, 1998). En ce sens, ces nouvelles immigrations ont un impact négatif sur l'identité européenne, car elles contribuent à maintenir le statu quo fondé sur la nation. Quelles que soient les constructions sociales pan-européennes que peuvent développer les migrants (l'islam, par exemple, a pris des formes spécifiquement européennes dans la vision de jeunes leaders tels que Tariq Ramadan), les pressions en matière de socialisation auxquelles doivent faire face les immigrants musulmans sont essentiellement nationales. Au cours des dernières années, presque tous les États nations en Europe ont élaboré des « politiques d'intégration " qui sont principalement le reflet des inquiétudes en matière de cohésion nationale sur la manière de transmettre la culture 
et les valeurs nationales aux nouveaux arrivants, mais il existe très peu des types de réponses post-nationales qui pourraient résulter d'une réelle européanisation des enjeux (Favell, 2003b). Le message le plus encourageant entendu par ces immigrants dans les sociétés d'accueil est "intégrez-vous, sinon...".

Ainsi, l'immigration est principalement une question nationale via la problématique de l'intégration. Même les mouvements progressistes et ouverts pensent d'inclusion dans les identités nationales: il s'agit de trouver sa place dans la Grande-Bretagne «multiethnique », ou dans la France " républicaine " ou aux Pays-Bas " tolérants et pluralistes ", etc. Comme le montrent les récents débats dans tous ces pays, les questions relatives à l'immigration et les populations vulnérables qui les incarnent sont constamment remises sur le tapis à la fois par les dirigeants politiques pro- et anti-immigration et par les médias dans de grands débats nationaux sur la citoyenneté, la culture nationale, l'acquisition de la langue et l'absorption dans le système d'aide sociale et le marché du travail nationaux. L'immigration non-européenne en cours est en fait devenue un des principaux moyens par lesquels se poursuit de manière classique l'affirmation de la nation dans l'Europe contemporaine, malgré les autres processus d'européanisation et de mondialisation qui sont à l'œuvre. Si par construction de l'Europe ou d'une identité européenne, on entend quelque chose se situant en sus et au-dessus de l'État nation (postnational, cosmopolite, l'émergence d'une "société » européenne, etc.) alors l'"Europe " n'est tout simplement pas pertinente pour la plupart des questions de politique d'immigration traditionnelles.

De plus, les réactions anti-immigrés observées en Europe avec le durcissement des politiques d'immigration et d'intégration sont également un pilier important de la réaction anti-UE. Elles constituent elles aussi un facteur distinct dans la déconstruction de l'identité européenne (au sens post-national) bien que, comme le souligne Douglas Holmes, ces politiques aient elles-mêmes entraîné une européanisation des nationalistes et des opposants à l'immigration dans leur propre vision différente de l'Europe, à savoir une Europe d'États nations (2000). Dans l'ensemble, les peuples restent hostiles aux migrations de tous types. Les politiques d'immigration progressistes (sur les droits à la citoyenneté, etc.) n'ont pu avancer politiquement en Europe que par la dépolitisation de ces questions en les plaçant sur les terrains juridiques et techniques, généralement pendant des périodes d'accalmie du débat 
politique autour des questions d'immigration (Guiraudon, 1998). Selon les termes de l'analyse de Neil Fligstein sur la manière et les raisons qui font que les personnes se sentent "européennes ", l'immigration fait en réalité partie des questions qui font que les ressortissants des États membres se sentent bien moins européens, y compris les immigrés euxmêmes qui n'ont d'autre choix, s'ils souhaitent être intégrés dans leur société d'accueil, que de se conformer aux pressions en matière d'intégration et de "nationalisation ", associée à l'acquisition de la citoyenneté et d'un statut officiel. Les effets de l'immigration dénotent une dynamique opposée à l'hypothèse de Deutsch : une plus grande mobilité et une interaction accrue aboutissent à une Europe moins intégrée.

Un point de vue différent sur les effets conjugués de la nouvelle immigration pourrait émerger en considérant la question en termes structurels et économiques, au lieu des termes culturels que l'on retrouve dans les débats politiques et les discussions autour de l'identité. Loin du maelström de la rhétorique des politiques intérieures, les décideurs ont certes abordé la coordination ou l'élaboration de politiques d'immigration au niveau de l'UE. Les tentatives d'externalisation de l'immigration et du contrôle aux frontières fondées sur la sécurité constituent d'ailleurs le seul champ de politique de l'UE dans lequel on assiste à une intégration rapide. Il s'agit plus pour les nations de déléguer efficacement les mécanismes de contrôle à une agence efficace qu'une entreprise supranationale en tant que telle, mais cela a assurément entraîné une européanisation des forces de police et d'autres agences d'État. Il semble y avoir un travail énorme de redirection du projet d'intégration européenne interne vers la construction et le renforcement des frontières extérieures (en particulier au Sud). Dans ce sens, l'immigration apparaît comme participant à la construction (négative) de l'Europe, comme " forteresse " telle qu'elle est souhaitée par des populations inquiètes dans les différentes nations.

Toutefois, l'économie pourrait mettre à mal cette vision particulière de l'Europe. Le nombre d'immigrés extracommunautaires augmente encore, malgré les tentatives de contrôle. Cette migration semble encore répondre à un besoin structurel en migrants, correspondant à des besoins en matière démographique dus au déclin de la natalité et au vieillissement de la population, ainsi qu'à la restructuration économique de l'État-providence national à l'européenne vers une économie de services mondialisée. Aucun État nation européen ne peut échapper à 
l'évolution structurelle du marché de l'emploi, à son élargissement à l'échelle européenne, organisé partout autour de pôles importants constitués par des «villes globales ». Dans chaque économie européenne, on assiste à une division du travail et à une augmentation des inégalités entre les secteurs principaux d'emploi des classes moyennes et supérieures, et les emplois moins qualifiés de la classe ouvrière que les autochtones sont de moins en moins nombreux à vouloir occuper : travail d'entretien, aide à domicile, employés dans la restauration, ouvriers d'usine, chauffeurs de taxi, ouvriers dans le bâtiment et l'agriculture, etc. Il existe une résistance nationale à ce processus, ainsi que des différences entre les "variétés de capitalisme européen " (Esping-Andersen, 1999). La Grande-Bretagne est beaucoup plus inégalitaire et par conséquent ouverte aux migrants que, par exemple, le Danemark. Cependant, ces effets économiques structurels se retrouvent dans tous les pays.

Ces processus participent ainsi à l'intégration de l'Europe selon une nouvelle rationalité économique. Avec leurs trajectoires économiques convergentes, les économies européennes finissent par ressembler de plus en plus à l'Amérique du Nord en ce qui concerne leur besoin structurel en migrations pour occuper les emplois du marché du travail secondaire : cela signifie pour l'avenir des niveaux importants de migrations tacites, la marginalisation sociale et économique des immigrés et des politiques aux frontières officiellement axées sur le contrôle, mais poreuses et libérales dans leurs effets; des politiques de contrôle aux frontières désignées dans le contexte américain de "miroir aux alouettes" (Massey et al., 2002). Vue sous cet angle, l'immigration participe encore à la construction de l'Europe, d'un point de vue structurel, même si les populations des pays d'accueil n'apprécient pas vraiment l'Europe en apparence néolibérale qui se construit par le biais de ces processus (Diez Medrano, 2009).

\section{Eurostars et Eurovilles}

Qu'advient-il lorsque vous enlevez les questions de couleur, de classe, d'origine ethnique, d'inégalité, de frontières, de barrières et de position de faiblesse culturelle de l'immigration ? Réponse : vous obtenez des "libres circulants». Les ressortissants des États membres de l'Europe sont également des citoyens européens, dont les droits incluent la possibilité de se déplacer, de consommer, de travailler, de vivre et de s'installer sans entrave où ils le souhaitent en Europe, quand ils le souhaitent et 
comme ils le souhaitent. À l'échelle mondiale, l'Union européenne est un espace unique de ce point de vue : il n'existe aucun autre espace post-national construit politiquement de ce type sur la planète. Les citoyens européens bénéficiant de la libre circulation n'ont pas besoin de visas. Ils n'ont à s'inquiéter ni de leur citoyenneté, ni de leur intégration. Souvent, ils n'ont même pas besoin d'avoir de résidence officielle pour vivre et travailler où ils le souhaitent (ils peuvent aller et venir librement dans l'espace européen). La législation européenne sur la libre circulation des personnes inscrite dans le traité de Rome de 1957 a défait la logique nationaliste des États nations comme communautés d'appartenance au moment où les migrations d'après-guerre et la mise en place des États-providence européens cimentaient fermement les systèmes nationaux.

Ensuite, en théorie, la libre circulation en Europe pouvait constituer une piste pour construire une Europe de type très différent. Toutefois, le nombre de migrants de ce type est resté faible historiquement. Aujourd'hui encore, moins d'un Européen sur cinquante vit hors de son pays d'origine et les chiffres n'ont augmenté de manière significative qu'au cours des étapes majeures vers l'intégration européenne. L'intérêt de cette population peu nombreuse de "libres circulants " par rapport aux migrants traditionnels réside précisément dans son caractère de cas limite inattendu : ils révèlent exactement ce que les autres types d'immigrants doivent faire pour être à pied d'égalité avec les ressortissants nationaux (le développement qui suit s'appuie sur Favell, 2008a).

Les «Eurostars» comme je le nomme sont au cœur des efforts de la Commission européenne pour construire une Europe via des politiques de mobilité. Aujourd'hui, il ne s'agit plus de déplacer des mineurs et des ouvriers du Sud vers le Nord, comme c'était le cas lorsque la liberté de circulation des personnes a été inscrite comme l'une des dispositions principales du Traité de Rome en 1957. Le propos de la stratégie de Lisbonne présentée à grands renforts de publicité en 2000 concerne plutôt les mouvements de personnes hautement qualifiées, à savoir la circulation des talents dans une économie de la connaissance, ayant pour effet secondaire la construction de l'identité européenne. Ce mouvement a de fortes chances de devenir un phénomène de concentration à dominante urbaine, d'où l'émergence d' "Eurovilles " constituant un réseau de lieux cosmopolites moteurs de la nouvelle économie européenne : des grandes villes comme Londres, Amsterdam, Bruxelles, 
Milan, Munich, Berlin, Barcelone, Vienne, entre autres, pas complètement ancrées dans les sociétés dans lesquelles elles se trouvent.

Londres a incontestablement été la plus grande bénéficiaire de ce phénomène depuis les années 1990. Au large du continent, hub mondial et passerelle pour l'ensemble de l'Europe, Londres est la destination par excellence de la libre circulation, avec une économie urbaine qui a attiré les plus brillants et les meilleurs éléments d'une génération entière de "libre circulants" français, allemands, italiens et espagnols (ainsi que d'autres nationalités), frustrés par la stagnation de l'économie et la rigidité provinciale des hiérarchies professionnelles dans leur pays d'origine. Ce qui a débuté comme une migration importante, mais plutôt invisible d'Européens de l'Ouest a pavé la route pour une nouvelle génération de jeunes polonais, hongrois et roumains talentueux et éduqués, et pour d'autres qui prennent la même direction, entre autres parce que d'autres pays (comme la France et l'Allemagne, en particulier) ont imprudemment fermé leurs portes à ces mêmes immigrants de premier choix des nouveaux pays membres. Ce type de mobilité européenne est également présenté comme modèle pour les migrations futures post-élargissement. Au minimum, le nombre des citoyens de l'UE originaires d'Europe de l'Est utilisant les opportunités offertes par l'Europe de devenir des « libres circulants " et non plus des " immigrés » devrait largement dépasser le nombre dérisoire d'Européens de l'Ouest qui a déménagé grâce à ces mêmes droits au cours des trois ou quatre décennies précédentes.

Ces " libres circulants ", pionniers de la vie d'Eurostars, sont sans aucun doute ceux qui ont le plus de chance de ressentir et de créer une identité européenne post-nationale/cosmopolite. Comme le décrit de manière détaillée l'étude PIONEUR (Recchi et Favell, 2009), ils incarnent en chair et en os (quoiqu'en petit nombre) ce dont les philosophes et les théoriciens du social cosmopolites rêvent depuis longtemps ${ }^{3}$. Ceci n'est pas surprenant en soi. Selon les termes de Fligstein, ces

3 Financé par le Cinquième Programme cadre de l'UE, le projet PIONEUR (20032006) "Pioneers of European Intergation "from below": Mobility and the Emergence of European Identity among National and Foreign Citizens in the EU », sous la direction d'Ettore Recchi de l'université de Florence, a conduit la première enquête systématique jamais menée sur les citoyens européens migrant au sein de l'UE, interrogeant environ 5000 résidents étrangers des cinq plus grands États (UE15) dans les cinq plus grands Lander (Allemands en Grande-Bretagne, en France, en Italie et en Espagne; 
personnes représentent sans aucun doute le prototype des Européens même si, comme le montre l'étude PIONEUR, ils rencontrent de petites difficultés à combiner leur nouvelle identité européenne avec des identités trouvant leurs racines à la fois dans leur pays d'origine et dans leur pays de résidence (Rother et Nebe, 2008).

Jouent-ils pour autant un rôle dans la construction ou l'évolution de l'Europe d'un point de vue macrostructurel ? L'ironie de la situation est évidente : en supprimant toutes les barrières, en créant une multitude de dispositifs incitatifs, en dépeignant une Europe sans frontières et offrant seulement des opportunités, on obtient en fait des niveaux de circulation statistiquement insignifiants. L'explication la plus courante consiste à dire que les Européens n'aiment pas bouger, qu'ils sont par nature enracinés dans la région où ils sont nés par la culture et par la langue. Mais cette explication par une vision culturaliste de l'Europe est intenable étant donné l'importance des migrations dan son histoire, et le fait que l'anglais est devenu une seconde langue courante qui est utilisée indifféremment par les Européens dans le cadre professionnel ou dans les entreprises multinationales implantées dans les grandes villes. Quelle que soit la cause de l'immobilité, les circulations transfrontalières des Européens constituent un moyen terriblement lent et marginal de construction de l'Europe. Tant pis pour la philosophie et la théorie économique. Ce scénario exceptionnel du " cas d'étude le plus probable " (" most likely case ») révèle toutefois les limitations réelles de tout espace postnational européanisé, même dans la situation théoriquement la plus probable.

Ainsi, être Européen pour ces libres circulants n'est certainement pas une chose banale en termes de questions relatives à l'identité. Il existe assurément des formes d'une nouvelle vie européanisée qui ont émergé, des espaces fortement européanisés au sein des villes avec une population de «nouveaux Européens ». Mais surtout, malgré les faibles chiffres, lorsque l'on étudie les vies, les expériences et les trajectoires de ces " citoyens du monde » en puissance d'un point de vue ethnographique, il apparaît que les circulants doivent faire face à des obstacles locaux et

Britanniques en Allemagne, en France, en Italie et en Espagne ; Français dans les quatre autres pays, etc.). Elle a posé des questions sur le profil socioéconomique et géographique de ces migrants, leurs motifs de migration, leur mobilité spatiale et sociale, leurs sentiments vis-à-vis de la citoyenneté européenne, leur consommation médiatique et leur participation politique. Voir aussi notre site web : http://www.obets.ua.es/pioneur. 
nationaux surprenants qui bloquent leurs aspirations, même dans les endroits les plus ostensiblement cosmopolites.

Après Londres, Amsterdam constitue une autre plateforme ou « hub» de la mobilité européenne. L'économie des Pays-Bas est souvent classée comme la plus ouverte du monde et Amsterdam est vue comme la capitale européenne des libertés, avec la culture urbaine considérée comme la plus ouverte et la plus tolérante du continent. C'est une destination très populaire parmi les migrants irlandais, britanniques, allemands ou originaires d'Europe du Sud. Mais là aussi, les ressortissants de pays de l'UE se retrouvent souvent exclus de manière informelle dans les lieux de résidence choisis par des processus d'exclusion particuliers, extrêmement ethnicisés. À Amsterdam comme dans d'autres Eurovilles, ces processus se caractérisent par une concurrence sans merci pour bénéficier des fruits de l'enracinement, c'est-à-dire de la meilleure qualité de vie urbaine dans les quartiers les plus prisés des villes globales. Les Néerlandais sont certes ouverts, tolérants, cosmopolites, mais ils ne facilitent pas la tâche au quotidien à ceux qui souhaitent s'installer, faisant en sorte que même leurs voisins européens les plus proches aient un accès minimal aux secrets intimes d'une "culture " nationale réservée aux personnes qui parle le néerlandais. Cette " culture » structure les traditions déroutantes et les règles qui régissent l'organisation de la vie quotidienne, l'accès aux meilleurs logements, à une qualité de vie, difficile à atteindre, obtenues après des années par les Amstelodamois grâce à une stratégie bien élaborée. Malgré son attrait et le grand nombre d'étrangers qui s'y installent, peu d'entre eux restent, le turnover est important et les Pays-Bas ont une des populations de résidents étrangers les plus faibles du continent. Un capital réservé à l'élite et l'adoption d'une vie d'" expatrié " déconnectée de la ville sont nécessaires pour surmonter ces barrières (stratification empêchant une massification de la mobilité européenne par le bas, concernant les classes moyennes, ou un impact de type structurel sur l'Europe des États nations). La mobilité restera exclusivement réservée aux élites, si tant est qu'elle se maintienne, et les opportunités de mobilité dans l'espace européen n'entraîneront ni nouvelle mobilité sociale, ni l'émergence d'une sensibilité cosmopolite plus répandue (voir aussi Recchi, 2009, pour une analyse quantitative des résultats de l'étude PIONEUR dans ce sens).

Il ne serait pas justifié de dire que l'espoir d'une construction de l'Europe par les Européens qui se déplaceraient et s'installeraient à long terme à l'étranger constitue un objectif un peu trop ambitieux. 
Cependant, ces mêmes Européens placent invariablement (dans les enquêtes de l'Eurobaromètre) leur droit à la libre circulation en tête des bénéfices importants que leur apporte l'appartenance à l'UE (devant l'économie et la sécurité) et environ un tiers déclarent être prêts à s'installer à l'étranger si l'opportunité et le besoin se présentaient (Année européenne de la mobilité des travailleurs 2006) ${ }^{4}$. D'autres formes de mobilité plus passagères/temporaires en Europe seront peut-être plus significatives : hommes d'affaires, étudiants, retraités, clients, commerçants transfrontaliers, navetteurs, etc. Toute expérience de mobilité transfrontalière, même sur le plus court terme, peut avoir des impacts importants en termes d'européanisation. En revanche, si la mobilité consiste principalement à prendre des vacances avec des compatriotes sur une plage de la Costa del Sol, ou à aller se déchaîner comme un hooligan lors d'un match de championnat d'Europe de football, elle ne débouchera pas sur une identité européenne. Jusqu'à présent, les constatations restent ambiguës, même pour les bénéficiaires les plus évidents comme les étudiants Erasmus ou les immigrés retraités. La plupart des observations sur ces autres formes de mobilité semblent montrer que l'expérience de ces bénéfices de l'Union européenne n'est ni particulièrement destructrice, ni transformatrice des cultures ou des identités nationales (comme le montre également l'étude PIONEUR ; voir aussi King, Warnes et al., 2000 ; King et Ruiz-Gelices, 2003), et que, lorsque les Européens rentrent chez eux, ils retournent vers leurs identités nationales principales. Les Eurostars, en revanche, essayent explicitement d'avoir une vie post-nationale; la plupart d'entre eux trouvent que ce n'est pas chose facile aujourd'hui dans l'Union européenne.

\section{Circulants Est-Ouest}

Le contraste entre ces deux types de migrants en Europe (migrants non-européens et ressortissants de l'UE appartenant à l' "élite ») souligne malheureusement l'écart en termes d'opportunités de mobilité devenu un cliché dans la littérature sur les vainqueurs et les perdants des

\footnotetext{
4 Parmi les personnes interrogées, $37 \%$ pensent qu'elles seraient prêtes à déménager dans un autre pays qui leur offrirait une meilleure situation, $53 \%$ pensent que la "liberté de voyager et de travailler dans l'UE » est le bénéfice principal de l'adhésion - devant l'euro (44\%) et la paix (36\%) - et $57 \%$ ont voyagé à l'étranger au sein de l'UE au cours des deux dernières années. Pourtant, moins de $4 \%$ ont déjà vécu et travaillé à l'étranger.
} 
processus de mondialisation et d'intégration régionale (voir par exemple Bauman, 1998). L'image est pourtant loin de refléter la réalité (sur ce sujet, voir Smith et Favell, 2006). Parmi les migrants non-européens, on compte un nombre conséquent d'individus issus des classes moyenne et supérieure hautement qualifiées, qui utilisent souvent le relatif capital social et économique qu'ils ont acquis dans leur pays d'origine pour obtenir un travail de niveau inférieur à l'Ouest, ce qui s'apparente à un déclassement social en contrepartie d'une meilleure rémunération. Il s'agit d'un fait universellement reconnu dans les migrations que ce ne sont pas les plus pauvres et les moins capables de migrer qui se déplacent, mais ceux qui disposent d'un certain niveau de capital humain ou d'un capital social basé sur des réseaux (Hammar et al., 1997). Parmi les migrants intracommunautaires, en attendant, il apparaît clairement que le droit de libre circulation en Europe a été utilisé de manière plus efficace par une nouvelle génération de "social spiralists " - migrants qui montent dans l'échelle sociale, ambitieux, provinciaux, travailleurs et de classe moyenne inférieure, souvent originaires du Sud, souvent de sexe féminin, utilisant la migration internationale en Europe comme moyen d'échapper aux frustrations liées à leur carrière professionnelle et leur style de vie dans leur lieu d'origine (Favell, 2008a, 64).

La caricature opposant migrants pauvres et « libres circulants » appartenant aux élites sociales est encore moins applicable à la nouvelle forme de migration qui s'est développée au cours des dernières années pour devenir la preuve la plus visible de l'évolution de l'Europe, à savoir la migration post-élargissement. Cette migration peut être datée après 2004 et 2007, même si la réalité était déjà bien établie auparavant, à la fois de manière informelle et semi-formelle, et concerne des ressortissants de pays qui n'appartiennent pas encore aujourd'hui aux vingt-sept pays officiellement membres de l'Union européenne'. La discussion qui suit concerne les ressortissants des dix pays de l'Europe centrale et

5 Les trois quarts des primo-arrivants dans l'Europe des 27 en 2006 venaient des nouveaux États membres: 1,2 million sur 1,6 million (Recchi et Triandafyllidou, 2009). Bien plus que 500,000 Polonais, par exemple, ont été enregistré comme vivant au Royaume-Uni entre 2004 et 2006. En fait $40 \%$ des citoyens des pays de l'Est qui ont demandé un permis de séjour en 2004 au Royaume-Uni y résidaient déjà (Recchi et Triandafillydou, 2009). Après 2007, le « stock " de ressortissants roumains et bulgares installés dans les autres pays de l'Union était pratiquement équivalent à celui des pays entrés dans l'UE en 2004: 1,7 million et 2,1 million de personnes respectivement - sachant qu'évidemment en proportion de la population totale des pays d'origine, les 
orientale (PECO), citoyens des pays qui ont récemment rejoint l'UE, et par extension les migrants de tous les pays candidats, potentiellement candidats, associés et voisins de l'UE à cause des effets territoriaux, "centrifuges " de l'intégration des marchés à l'échelle régionale via la "Politique européenne de voisinage ( Rogers, 2000 ; Favell, 2008b). La question est de savoir si les individus de la migration post-élargissement suivent la trajectoire classique des immigrés non-européens, ou s'ils sont plutôt des " libres circulants " amenés à devenir les libres citoyens européens du futur ? Quel est le scénario qui s’applique le mieux ?

Les théories démographique et économique sont ici très encourageantes. Les nouveaux " libres circulants " incarnent le triomphe de la théorie de l'intégration européenne, qui prédit une issue gagnantgagnant-gagnant pour l'Europe à cause de ces nouveaux mouvements (ECAS, 2005, 2006) : les migrants sont gagnants car ils satisfont leurs ambitions et leurs objectifs de mobilité, la société d'accueil est gagnante car elle reçoit les bénéfices de leur talent et de leur enthousiasme et la société d'origine bénéficiera de la dynamique de développement positive initiée par les mouvements d'individus talentueux qui font circuler les devises et les réseaux dans la nouvelle Europe, et qui rapporteront leurs talents à un moment donné dans l'économie de l'Est nouvellement intégrée (où se trouveront de meilleures opportunités dans le futur). En résumé, les mouvements promeuvent théoriquement l'intégration européenne, une dynamique économique efficace et une circulation des compétences et du capital qui se transformeront en retour en développement. Confirmant cette théorie, on assiste à une mobilité spatiale/ sociale accrue parmi les circulants originaires d'Europe de l'Est. Cette génération de nouveaux Européens est ambitieuse, avec des « libres circulants » dynamiques prêts à obtenir ce qui leur appartient de l'Ouest, tout en tirant avantage de la possibilité d'aller et venir entre l'Ouest et l'Est. Plus d'un demi-million de ressortissants des huit PECO ayant rejoint l'Union en 2004, par exemple, ont tenté leur chance dans l'économie florissante britannique depuis 2004. Sur ce point au moins, ils participent sans aucun doute à la construction de l'Europe (indépendamment de ce qu'ils expriment ou de ce que les autres expriment dans les Eurobaromètres ou

flux de roumains et bulgares sont plus importants (Recchi et Triandafyllidou, 2009). En tout état de cause, les chiffres sous-estiment le nombre de travailleurs des PECO puisqu'ils ne comptent pas les saisonniers, les travailleurs temporaires et les frontaliers. 
en termes d'identité). Ce mouvement constitue sans doute le plus grand changement social des cinquante dernières années en Europe : la fin définitive de la guerre froide et une expérience sociale européenne qui ne laissera intacte ni l'Est ni l'Ouest.

Toutefois, dans le même temps, un scénario politique négatif se poursuit en réaction à ces migrants considérés comme des "immigrés " plutôt que comme des « libres circulants ». L'identité peut être mobilisée de manière négative sur cette question si les migrants sont considérés par les populations des pays d'accueil comme des menaces plutôt que comme des soutiens bienvenus pour les économies nationales. Les éléments observés sur ce point sont équivoques. Globalement, la plupart de ces migrants ont fait l'objet de réactions pragmatiques. Pour les Roumains à Milan et Bilbao, les Polonais à Berlin et Londres, les Russes et Yougoslaves à Paris ou Amsterdam, la réaction politique n'a pas atteint la même violence que celle subie par les populations immigrées (relativement) plus visibles originaires d'Afrique du Nord et d'Afrique noire dans les mêmes pays. Certes, le plombier polonais et l' "invasion " de Rome ont fait la une des journaux en France et en Grande-Bretagne ; la France reste pratiquement fermée aux ressortissants des nouveaux pays membres $^{6}$ et la Grande-Bretagne a fermé ses portes aux Roumains et aux Bulgares. Cependant, la réaction a été globalement lente et mesurée, plus en phase avec la prise de conscience progressive que ces nouveaux visages font plutôt partie d'un système économique Est-Ouest amené à durer, et qui pourrait avoir des conséquences bien plus importantes que la simple installation de nouveaux immigrants " ethniques".

Cette réaction peut survenir même lorsque ces migrants sont Blancs, chrétiens, compétents et répondent à un fort besoin (et cela peut concerner autant l'ouvrier agricole roumain en Italie que le commerçant polonais en Allemagne ou encore l'employé de bureau hongrois très compétent en Angleterre [voir les ethnographies dans Favell et Elrick 2008]). L'ambiguité de leur présence est accentuée par le fait qu'il n'est pas si évident que les formules traditionnelles en matière d'intégration inspirées de la formation des États nations s'appliquent ici. Les populations immigrées lors des vagues précédentes pourraient être contrariées par le

6 Les ressortissants des PECO comme les Polonais doivent obtenir de haute lutte un permis de travail "étranger ". Les restrictions sur l'accès au marché du travail des citoyens des PECO devraient prendre fin en 2011. 
fait que les Européens de l'Est puissent aller et venir, occuper les mêmes emplois, sans être soumis à aucune pression en matière d'intégration, alors que les migrants non-européens doivent démontrer leur " appartenance » aux pays d'accueil d'Europe occidentale, notamment via des cours ou des contrats d'intégration ou des parcours de citoyenneté. Il reste possible d'incorporer ces migrants dans les processus traditionnels d'exclusion/inclusion mais, pour l'essentiel, les nouvelles dynamiques migratoires Est-Ouest définissent des schémas de mobilité pendulaire, temporaire et non résidentielle dans le nouvel espace européen (Morawska, 2002; Wallace et Stola, 2001). Les populations locales sont horrifiées lorsque les épouses et les enfants des ouvriers polonais encombrent les institutions scolaires et médicales. Ils ont bien compris que la solution consiste à s'assurer d'avoir des liaisons aériennes low cost suffisamment nombreuses, de faciliter la mobilité et de garantir une situation économique prometteuse dans les pays d'origine pour que leur installation soit temporaire. En restant ouvertes et suffisamment poreuses, les nations d'Europe de l'Ouest pourraient mettre en œuvre la formule adéquate avec la Pologne, mais la poursuite de la fuite des cerveaux et des bras constitue actuellement un danger pour les nouveaux États membres de la région des Balkans.

Pour évaluer l'avenir de ce nouveau système de migration européen, il est donc nécessaire de s'interroger sur le type d'Europe qu'il est en train de construire. L'intégration de l'Europe de la mobilité promise par les démographes et les économistes est effectivement en train de se produire. Mais le système vers lequel on tend est dans la plupart des cas un système basé sur un marché du travail dual dans lequel les Européens de l'Est occuperont des rôles secondaires, temporaires, flexibles fondés sur la possibilité d'exploitation en termes d'avantages de coût et de capital humain. Ainsi, l'Europe en vient à ressembler au modèle constitué par les États-Unis, où les circulants Est-Ouest occupent les emplois les plus durs et dangereux ou se heurtent à des barrières invisibles, et où les distinctions " ethniques » entre l'Est et l'Ouest ont peu de chances de disparaître. Dans un certain sens, ce mode d'inclusion perpétue la longue relation historique inique entre Est et Ouest. Les Européens de l'Est pourront circuler et ils feront l'amère expérience que l'Ouest ne les accepte que pour les emplois dont les Occidentaux ne veulent pas. En résumé, le danger est qu'ils deviennent une nouvelle classe de domestiques au sens victorien pour une aristocratie de mères d'Europe 
de l'Ouest éduquées dans les universités et de « créatifs ", qui ont besoin d'aide pour mener à bien leurs vies de rêve.

La logique centrifuge de l'intégration régionale pousse dans le même temps l'UE à engager des négociations au-delà de ses frontières orientales, en gérant des scénarios particuliers aux frontières avec les nouveaux voisins pour encourager les échanges commerciaux et la mobilité tout en gardant le contrôle sur les implications indésirables en matière de sécurité et de surveillance. Une nouvelle fois, l'idée sous-jacente est que tous les types de libre circulation peuvent être gérés, et que de leur bonne gestion dépend la situation de gagnant-gagnant pour les deux parties. Cette caractéristique de l'intégration européenne place l'Europe en avance par rapport aux autres entreprises d'intégration régionale existant dans d'autres parties du monde. Grâce à des accords bilatéraux avec ses voisins extérieurs et des politiques de voisinage, le projet d'intégration européenne s'étend loin à l'Est jusqu'à l'Oural, au Sud jusqu'aux monts de l'Atlas et englobe les régions jusqu'aux frontières de la mer Morte. Il est probable que les mouvements de population de tous types se développeront dans cet espace européen et que ceux qui s'y déplacent réclameront de nouveaux droits en tant que libres circulants européens de facto plutôt qu'en tant qu'immigrants non-européens de jure.

\section{Conclusion : Migration et processus d'intégration régionale comparés}

Les différents types de migration participent à la construction de l'Europe selon de nouvelles modalités, mais c'est le nouveau système de migration engendré par les élargissements successifs de l'UE et les autres initiatives liées à la politique européenne de voisinage qui devrait être d'une importance capitale. Alors que la logique de l'élargissement l'emporte sur celle de l'approfondissement, un nouvel espace régional européen est en cours de construction, réglant les questions de démographie/ économie/ sécurisation (même si les frontières de cet espace ne se limitent assurément pas aux frontières de l'UE à 27). Au moment où l'intégration politique de l'UE se heurte à un mur, toute l'énergie constructive de l'UE a été reportée sur les initiatives de maîtrise des frontières poreuses d'un nouvel espace régional, ouvrant ainsi la mobilité et les quatre libertés (au moins de façon partielle) à une UE à 45 ou 55 par le biais de l'extension du marché européen au-delà des conceptions antérieures de l'Europe. 
La question clé sera bien évidemment de situer les frontières de cet " espace " et de ce "système ». Qui se situe, à l'intérieur ? À l'extérieur ? En d'autres termes, quelle sera la relation entre les nouveaux " libres circulants " d'Europe de l'Est (à la fois des nouveaux pays membres et des futurs États membres éventuels), qui se déplacent de manière fluide dans l'espace européen, avec ces « immigrants non-Européens " postcoloniaux qui occupaient les emplois secondaires en Europe par le passé ?

Une manière de répondre à cette question consiste à revenir à la comparaison entre les États-Unis et l'Europe et à établir les grandes lignes de l'espace régional européen émergent par rapport à celles de l'espace régional nord-américain. Sous cet angle, il devient instructif de comparer la logique d'extension explicitement politique de l'intégration régionale européenne au scénario politiquement non maîtrisé et socialement désastreux auquel les États-Unis sont aujourd'hui confrontés avec l'Amérique centrale. Là où l'Europe se tourne de plus en plus vers l'Est pour ses besoins en main-d'œuvre de migrants, les États-Unis dépendent de la migration massive de Latino-Américains en provenance du Sud pour remplir les mêmes rôles. Et comme le montre la politique d'immigration récente aux États-Unis, le scénario de l'immigration entre le Mexique et les États-Unis (et par extension les relations avec l'ensemble de l'Amérique centrale) est incapable de prendre les mesures politiques efficaces vers une intégration régionale post-nationale à l'image de celle de l'Europe, tout en restant structurellement dépendants de la mobilité transfrontalière (Massey et al., 2002). L'Amérique a désespérément besoin d'une solution économique rationnelle, mais celle-ci est impossible pour des questions de politique intérieure.

L'économie et la société américaines continuent d'avoir besoin de l'immigration. L'économie américaine a plus que jamais besoin de frontières poreuses pour son fonctionnement. Sans cela, l'agriculture californienne s'effondrerait. Il en est de même pour le Texas. Que font les habitants de Los Angeles lorsqu'ils ont un problème de toiture, des soucis avec le système d'arrosage de leur jardin ou une clôture à garder la nuit? Ils appellent Carlos, un Américain d'origine mexicaine récemment naturalisé, qui vit en fait à Los Angeles depuis vingt-cinq ans. Celui-ci appelle alors quelques "gars " qu'il connaît et vous arrêtez de poser des questions. Ou si vous avez besoin d'aide pour nettoyer la maison ou garder les enfants, la femme de ménage de votre voisin, Susana, a toujours une "sœur » ou une " cousine " prête à venir contre 
quelques billets donnés de la main à la main. Les classes moyennes et supérieures peuvent ainsi se consacrer à leurs activités consistant à faire de l'argent en vendant des séries télévisées pour le câble ou à aller à leurs cours de yoga après avoir déposé les enfants à l'école. C'est ainsi que la ville fonctionne, que tout le monde gagne de l'argent et libère du temps pour soi. De nos jours, New York, Chicago, Atlanta et Houston fonctionnent exactement de la même manière et ce mode de fonctionnement se généralise à Dalton, en Géorgie, à Las Vegas, au Nevada, ou même (très bientôt) à Des Moines, dans l'Iowa. Les classes moyennes européennes de Londres, Paris ou Rome suivent la même logique économique et les aides domestiques auxquelles elles ont recours, et dont elles sont de plus en plus dépendantes, sont de plus en plus souvent originaires d'Europe de l'Est.

Pourtant, les deux continents divergent politiquement. L'hostilité aux immigrants progresse aux États-Unis, basée sur des arguments culturels et linguistiques non fondés témoignant d'une ignorance crasse de la dynamique des populations à l'œuvre dans les grands centres urbains (Huntington, 2004). Le Congrès a récemment échoué plusieurs fois à voter des textes visant à régulariser la situation des migrants sanspapiers et à gérer les besoins économiques en matière de travailleurs en allant vers un contrôle politique accru. Seuls le contrôle aux frontières et la sécurité intérieure ont été renforcés. Mais comme l'a bien montré un récent film satirique, "Un jour sans Mexicains " ( $A$ day without a Mexican) entraîne la paralysie du miracle de l'économie américaine. Le scénario du film s'est réalisé le jour des manifestations de masse qui se sont déroulées à Los Angeles et ailleurs au printemps 2006. L'Amérique a essayé de se tourner vers l'Est pour satisfaire ses besoins énormes en immigrants (on peut supposer que c'est précisément la raison pour laquelle les États-Unis sont si souples concernant l'immigration en provenance de Corée, du Japon, de Chine et du Vietnam), mais elle continue d'avoir besoin de Carlos et de son équipe. Les suprémacistes blancs sont passés de mode aux États-Unis, grâce à la démographie. Même la présidence Bush a compris qu'un républicain sans électeurs asiatiques ou latinos était désormais voué à l'échec électoral. Les personnes hostiles à l'immigration continueront à vouloir des barrières toujours plus hautes et ils pourront encore se sentir autorisés et légitimes à prendre les armes comme les miliciens (minutemen) patrouillant dans le désert de l'Arizona. Mais comme le montrent les camions qui affluent 
vers le nord par cette frontière, alors que le capital et les touristes américains prennent la direction inverse vers le Sud, l'économie des États-Unis ne s'arrête pas et ne s'arrêtera jamais à El Paso ou Tijuana - et une partie bien plus importante de l'Amérique centrale et de l'Amérique du Sud que ne voudraient l'admettre les citoyens américains fait sans aucun doute partie de l'Amérique du Nord (voir également Pastor, 2001). Mais, comme toujours, la compréhension politique est en retard sur cette réalité guidée par des facteurs économiques.

L'Europe est confrontée à des questions similaires en matière d'immigration, mais elle a choisi d'emprunter une voie différente politiquement et économiquement. Au XXe siècle, la grande faiblesse de l'Europe par rapport aux États-Unis résidait dans ses frontières mal établies et ses déchirements politiques et économiques internes (au prix de millions de morts). Les États-Unis étaient un État nation unique en son genre, pratiquant essentiellement une politique de portes ouvertes à l'immigration sans barrière interne aux migrations qui alimenta sa croissance et sa puissance. L'Europe a également fini par ouvrir ses sociétés à l'immigration et aux migrations intra-régionales et cherche maintenant à mettre en œuvre un scénario proche dans son dynamisme et dans l'abolition des contrôles intra-européens que celui qui domine les débats en Amérique du Nord. Londres, la ville qui symbolise le mieux le développement de la mobilité européenne fait maintenant de l'ombre au New York mythique des immigrants en termes de croissance de la population par les immigrants sur la période 2000-2005. Mais il existe une différence essentielle entre les continents. Les États-Unis ont dû se tourner vers le Sud pour trouver une main-d'œuvre bon marché. L'Europe, de son côté, peut se tourner vers l'Est, et c'est ce qu'elle fait de plus en plus.

Mais quelles sont alors les conséquences pour le Sud traditionnellement en relation avec l'Europe ? En d'autres termes, on retrouve à nouveau ici la question ethnique de l'identité européenne, le problème essentiel consistant à déterminer si les Marocains et les Turcs (qui constituent de loin les populations d'immigrants les plus nombreuses en Europe, et les étrangers les plus proches) sont également des Européens mobiles selon la logique de l'intégration régionale. La politique actuelle ne semble pas aller dans le bon sens : l'Europe pourrait choisir de tenir compte de l'hostilité aux immigrants et de traiter les Marocains et les Turcs de la même manière lamentable dont les États-Unis traitent leur force de travail " étrangère " en provenance d'Amérique centrale. Toutefois, par une 
logique purement économique/concentrique d'intégration régionale, leur mobilité pourrait finir par ressembler de moins en moins à la trajectoire traditionnelle de l'immigrant, nationalisante et intégrationniste, mais plus à celle des ressortissants des nouveaux pays membres et candidats à l'adhésion d'Europe de l'Est. D'autres peuples voisins au Sud et à l'Est pourraient également exprimer cette même revendication (bien que l'espace du voisinage européen, comme tout espace, doive se terminer quelque part). Les frontières physiques ont leur importance ; il est possible jusqu'à un certain point de construire des murs et de surveiller les mers. Mais d'autres mécanismes peuvent rendre la migration beaucoup plus désagréable. Si l'Europe pouvait supprimer sa dépendance vis-à-vis des travailleurs "ethniques " pour occuper les emplois moins qualifiés, subalternes de son économie ; si le besoin luimême pouvait être supprimé par une logique raciale et ethnocentrique? Pour le moment, cela semble être la logique sous-jacente fixée pour déterminer qui peut bénéficier ou non de la "libre circulation". L'extension de l'Europe à l'Est est souvent soupçonnée d'être basée sur le choix d'une logique ethnique, dans laquelle les frontières s'ouvrent à l'Est alors qu'elles se ferment au Sud. Cette conception est fonctionnelle du point de vue politique, intéressante économiquement et constitue une solution dynamique à court terme.

L'élargissement européen et les accords avec les pays du voisinage européen ont permis à l'Europe de se tourner vers l'Est et à surveiller les frontières au Sud (renforcement des frontières et soutien financier et politique, dans le cadre de politiques de voisinage, aux États du Sud en Afrique et au Proche-Orient, contre la mise en œuvre de moyens plus efficaces de contrôle à distance). La contrepartie pour une Europe qui exprime plus de méfiance envers ses voisins musulmans de ses frontières sud que des peuples balkaniques à ses frontières orientales. Les Roumains et les Ukrainiens sont blancs et chrétiens, contrairement aux Turcs, Marocains, Sénégalais ou Somaliens. Mais il y a un problème dans cette logique, à savoir qu'elle ne résoudra pas la question démographique sur le long terme. Les taux de natalité des Européens de l'Est sont aussi faibles que ceux de leurs voisins d'Europe de l'Ouest. La tension entre porosité à l'Est et fermeture au Sud a ainsi de fortes chances d'être une des caractéristiques centrales de l'intégration régionale en cours par le biais de l'élargissement. Comme pour toute analyse des mouvements de population, ce macro-processus très important peut être observé et compris dans sa dimension humaine par le biais des expériences des différents migrants. 


\section{RÉFÉRENCES BIBLIOGRAPHIQUES}

Andall Jacqueline (1998), "Catholic and State Constructions of Domestic Workers: The Case of Cape Verdean Women in Rome in the 1970s ", in Khalid Koser et Helma Lutz (dir.), The New Migration in Europe, Londres, Macmillan Press, p. 124-142.

Anderson Benedict (1991), Imagined Communities, Londres, Verso.

Bade Klaus (2000), Europa in Bewegung: Migration vom späten 18. Jahrhundert bis zur Gegenwart, Münich, Beck Verlag.

Batalova Jeanne et B. Lindsay Lowell (2006), " "The Best and the Brightest" : Immigrant Professionals in the US ", in Michael P. Smith et Adrian Favell (dir.), The Human Face of Global Mobility, New Brunswick, NJ, Transaction Publishers, p. 81-102.

Bauman Zygmunt (1998), Globalization: The Human Consequences, Cambridge, Polity Press.

Benton Gregor et Frank Pieke (dir.) (1998), The Chinese in Europe, Londres, Macmillan.

Bousetta Hassan (2000), « Political Dynamics in the City. Citizenship, Ethnic Mobilisation and Socio-Political Participation: Four Case Studies ", in Sophie Body-Gendrot et Marco Martiniello (dir.), Minorities in European Cities: The Dynamics of Social Integration and Social Exclusion at the Neighbourhood Level, Londres, Macmillan.

Brubaker Rogers (1996), Nationalism Reframed: Nationhood and the National

Question in the New Europe, Cambridge, Cambridge University Press.

Byrnes Timothy et Katzenstein Peter (dir.) (2006), Religion in an Expanding Europe, Cambridge, Cambridge University Press.

Castles Stephen et Mark Miller (2003) [3 éd.], The Age of Migration: International Population Movements in the Modern World, Londres, Palgrave Macmillan.

Diamond Jared (1997), Guns and Germs and Steel: The Fates of Human Societies, New York, W.W. Norton \& Co.

Diken Bülent (1998), Strangers, Ambivalence and Social Theory, Aldershot, Ashgate.

Esping-Andersen Gösta (1999), The Social Foundations of Post-Industrial Society, Oxford, Oxford University Press.

European Citizenship Action Service (2005), Who's Afraid of EU Enlargement?, Bruxelles, ECAS.

European Citizenship Action Service (2006), Who's Still Afraid of EU Enlargement?, Bruxelles, ECAS.

European Foundation for the Improvement of Living and Working Conditions (2006), Mobility in Europe: Analysis of the 2005 Eurobarometer Survey on Geographical and Labour Market Mobility, Dublin. 
European Year of Workers' Mobility (2006), Europeans and Mobility: First Results of an EU-wide Survey, Bruxelles, Commission européenne.

Eurostat (2009), L'Europe en chiffres, annuaire 2009, Luxembourg, Office des publications officielles des Communautés européennes.

Favell Adrian (2001), "Integration Policy and Integration Research in Western Europe: A Review and Critique ", in Alex Aleinikoff et Doug Klusmeyer (dir.), Citizenship Today: Global Perspectives and Practices, Washington, DC, Brookings Institute, p. 349-399.

Favell Adrian (2003a), "Games without Frontiers? Questioning the Transnational Social Power of Migrants in Europe ", Archives européennes de Sociologie, vol. XLIV, $\mathrm{n}^{\circ}$ 3, p.106-136.

Favell Adrian (2003b), "Integration Nations: The Nation State and Research on Immigrants in Western Europe ", Comparative Social Research, $\mathrm{n}^{\circ} 22$, novembre, p. 13-42.

Favell Adrian (2008a), Eurostars and Eurocities: Free Movement and Mobility in an Integrating Europe, Oxford, Blackwell.

Favell Adrian (2008b), "The New Face of East-West Migration in Europe ", Journal of Ethnic Migration Studies, vol. 34, $\mathrm{n}^{\circ} 5$.

Favell Adrian et Tim Elrick (dir.) (2008), "The New Face of East-West Migration in Europe ", Édition spéciale, Journal of Ethnic and Migration Studies, vol. 34, n 5 .

Favell Adrian et Randall Hansen (2002), « Markets against Politics: Migration,

EU Enlargement and the Idea of Europe ", Journal of Ethnic and Migration Studies, vol. 28, n 4, p. 581-601.

Guiraudon Virginie (1998), "Citizenship Rights for Non-Citizens: France, Germany and the Netherlands ", in Christian Joppke, Challenge to the Nation State, Oxford, Oxford University Press, p. 272-319.

Hammar Tomas, Grete Brochmann, Kristof Tamas et Thomas Faist (dir.) (1997), International Migration, Immobility and Development, Oxford, Berg.

Hansen Randall (1998), "A European Citizenship or a Europe of Citizens? Third Country Nationals in the EU ", Journal of Ethnic and Migration Studies, vol. 24, $\mathrm{n}^{\circ}$ 4, p. 751-768.

Hatton Tomothy J. and Jeffrey G. Williamson (1998), The Age of Mass Migration, Oxford, Oxford University Press.

Held David, McGrew Anthony, Goldblatt David et Perraton Jonathan (1999), Global Transformations: Politics, Economics and Culture, Oxford, Polity Press. Hobsbawm Eric (1987), The Age of Empire, New York, Pantheon Books.

Hobsbawm Eric (1983), The Invention of Tradition, Cambridge, Cambridge University Press.

Holmes Douglas (2000), Integral Europe: Fast-Capitalism, Multiculturalism, Neofascism, Princeton, Princeton University Press. 
Huntington Samuel (1996), The Clash of Civilizations and the Remaking of the New World Order, Chicago, Chicago University Press.

Huntington Samuel (2004), Who Are We? The Challenges to American National Identity, New York, Simon and Schuster.

Joppke Christian et Morawska Ewa (dir.) (2003), Toward Assimilation and Citizenship: Immigrants in Liberal Nation States, Londres, Palgrave Macmillan. Kastoryano Riva (1993), Etre Turc en France, Paris, LHarmattan.

King Russell (2002), "Towards a New Map of European Migration ", International Journal of Population Geography, vol. 8, $\mathrm{n}^{\circ}$ 2, p. 89-106.

King Russell, Warnes Tony et Williams Allan (2000), Sunset Lives: British Retirement Migration to the Mediterranean, Oxford, Berg.

King Russell et Ruiz Gelices Enric (2003), "International Student Migration and the European "Year Abroad" : Effects on European Identity and Subsequent Migration Behaviour ", International Journal of Population Geography, vol. 9, p. 229-252.

Koser Khalid et Lutz Helma (dir.) (1998), The New Migration in Europe. Londres, Macmillan.

Lesthaege Ron (dir.) (2000), Communities and Generations: Turkish and Moroccan Populations in Belgium, Bruxelles, VUB Press.

Mann Michael (1993), The Sources of Social Power, vol. 2. The Rise of Classes and Nation-States 1760-1914, Cambridge, Cambridge University Press.

Mann Michael (2005), The Dark Side of Democracy, Cambridge, Cambridge University Press.

Massey Douglass, Durand Jorge et Malone Nolan (2002), Beyond Smoke and Mirrors: Mexican Migration in an Era of Economic Integration, New York, Russell Sage.

Mazower Mark (1998), Dark Continent: Europe's Twentieth Century, Londres, Penguin.

Milward Alan (2005) [2e éd.], The European Rescue of the Nation State, Londres, Routledge.

Moch Leslie Page (2003) [2e éd.], Moving Europeans: Migration in Western Europe Since 1650, Bloomington, Indiana University Press.

Morawska Ewa (2002), "Transnational Migration in the Enlarged European Union: A Perspective from East and Central Europe ", in Jan Zielonka (dir.), Europe Unbound, Oxford, Oxford University Press, p. 161-190.

Pastor Robert A. (2001), Toward a North American Community: Lessons from the Old World for the New. Washington, DC, Peterson Institute.

Peri Giovanni (2005), "Skills and Talent of Immigrants: A Comparison between the European Union and the United States ", Institute of European Studies Working Paper, 050304, Berkeley, University of California. 
Phalet Karen, van Lotringen Claudia et Entzinger Hans (2000), Islam in de multiculturele samenleving, Utrecht, ERCOMER.

Piore Michael (1989), Birds of Passage: Migrant Labor in Industrial Societies, Cambridge, Cambridge University Press.

Recchi Ettore (2006), " From Migrants to Movers: Citizenship and Mobility in the European Union ", in Michael P. Smith et Adrian Favell (dir.), The Human Face of Global Mobility, p. 53-77.

Recchi Ettore (2009), "The Social Mobility of Mobile Europeans ", in Ettore Recchi et Adrian Favell (dir.), Pioneers of European Integration, Cheltenham, Edward Elgar.

Recchi Ettore et Favell Adrian (dir) (2009), Pioneers of European Integration, Cheltenham, Edward Elgar.

Recchi Ettore et Triandafillydou Anna (2009), The Changing Face of Intra-EU Migration after the Union's 2004 and 2007 enlargements, Communication au IXe congrès de la European Sociological Association, Lisbonne, 4 septembre.

Riccio Bruno (2001), « From "Ethnic Group” to "Transnational Community”: Senegalese Migrants' Ambivalent Experiences and Multiple Trajectories ", Journal of Ethnic and Migration Studies, vol. 27, $\mathrm{n}^{\circ} 4$, p. 583-599.

Rogers Alisdair (2000), "A European Space for Transnationalism? ", Transnational Communities Programme Working Paper, Series WPTC-2K07, Oxford, COMPAS. http://www.transcomm.ox.ac.uk/working \% 20papers/rogers. pdf.

Rother Nina et Nebe Tina (2009), "More Mobile, More European? Free Movement and EU Identity ", in Ettore Recchi et Adrian Favell (dir.), Pioneers of European Integration: citizenship and mobility in the EU, Cheltenham, Edward Elgar.

Sassen Saskia (2001) [2e éd.], The Global City, Princeton, Princeton University Press.

Smith Michael P. et Favell Adrian (dir.) (2006), The Human Face of Global Mobility: International High Skilled Migrants in Europe, North America and the Asia Pacific, New Brunswick, NJ, Transaction Press.

Torpey John (2000), The Invention of the Passport, Cambridge, Cambridge University Press.

Wallace Claire et Stola Dariusz (dir.) (2001), Patterns of Migration in Central Europe, Londres, Palgrave. 